C-A/AP/\#417 Jan. 2011

\title{
Gatling Gun: High Average Polarized Current Injector for eRHIC
}

\author{
V. N. Litvinenko
}

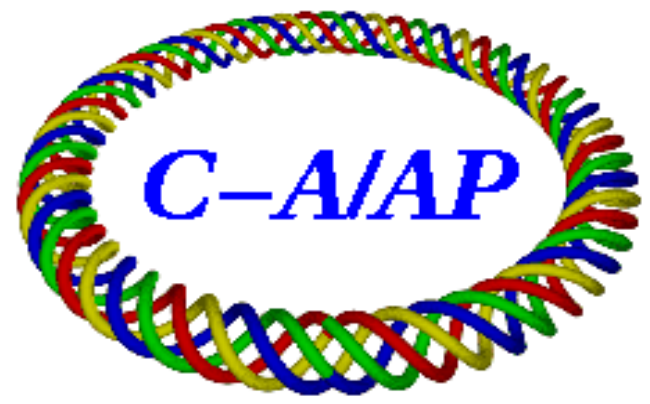

Collider-Accelerator Department Brookhaven National Laboratory Upton, NY 11973

Notice: This document has been authorized by employees of Brookhaven Science Associates, LLC under Contract No. DE-AC02-98CH10886 with the U.S. Department of Energy. The United States Government retains a non-exclusive, paid-up, irrevocable, world-wide license to publish or reproduce the published form of this document, or allow others to do so, for United States Government purposes. 


\section{Gatling Gun: \\ High Average Polarized Current Injector for eRHIC}

Vladimir N. Litvinenko

\section{Abstract.}

This idea was originally developed in 2001 for, at that time, an ERL-based (and later recirculating -ring) electron-ion collider at JLab. Naturally the same idea is applicable for any gun requiring current exceeding capability of a single cathode. ERL-based eRHIC is one of such cases. This note related to eRHIC was prepared at Duke University in February 2003.

\section{Introduction.}

In many case photo-injectors can have a limited average current - it is especially true about polarized photo-guns. It is know that e-RHIC requires average polarized electron current well above currently demonstrated by photo-injectors - hence combining currents from multiple guns is can be useful option for eRHIC.

\section{Idea of Revolver (or Gatling) Photo-gun.}

In a possible scenario when a single laser photo-gun is incapable of generating average current sufficient for eRHIC, we can use multiple guns operating with reduced rep-rate and combine their beams before injecting them into ERL. Fig.1 shows a sketch of such a system based on an RF cavity with rotating transverse mode. Transverse mode rotates with $f_{\text {rot }}=f_{e} / n$, where $f_{e}$ is the rep-rate of e-bunches in ERL. $n$ guns are located on a circle with $360^{\circ} / \mathrm{n}$ separation and generate pulses with rep-rate $f_{\text {rot }}$ and a $\Delta T=1 /$ fe time delay from each other. The beams recombine by the transverse kick in the cavity onto the injection axis of the ERL. Sketch shows "revolver" combiner (a Gatling gun) with $n=6$. If necessary, the cavity can be split in two with a horizontal and vertical transverse mode, shifted in phase by 90 degrees.

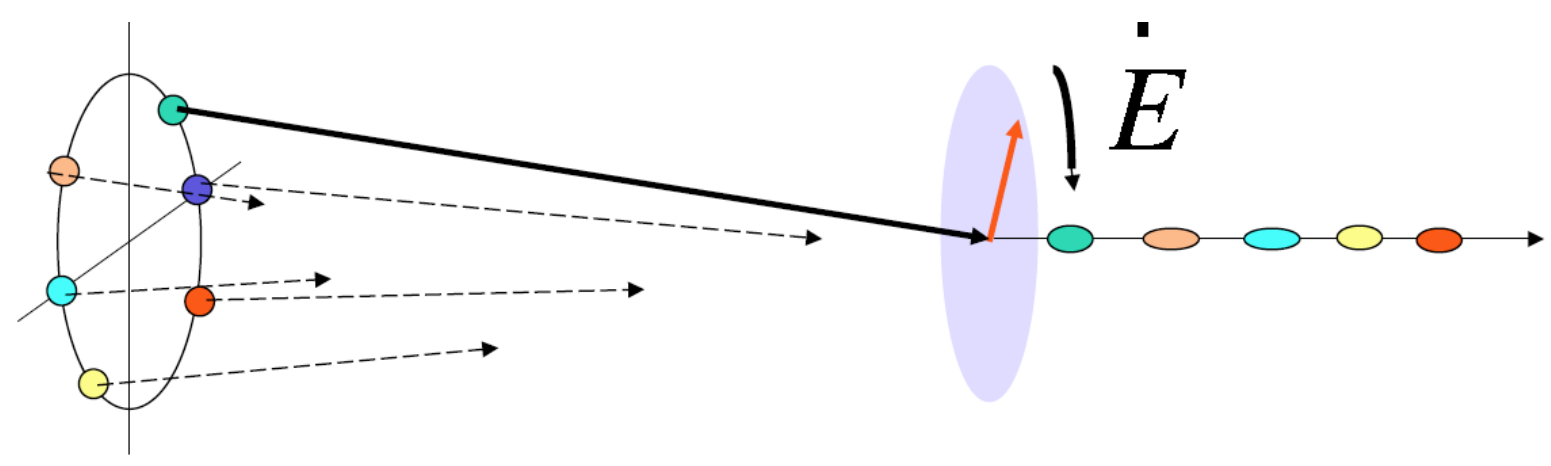

Fig. 1. RF-combiner for $\mathbf{n}$ photo-guns with one RF cavity.

The RF scheme is not the only one. The other possibility is the use of transverse kickers with horizontal kick $\theta_{x}=r / L \cdot \cos \left(2 \pi f_{r o t} t\right)$ and vertical kick $\theta_{y}=r / L \cdot \sin \left(2 \pi f_{r o t} t\right)$, where $\mathrm{r}$ is radial separation of the guns and $\mathrm{L}$ is the distance to the combiner. 


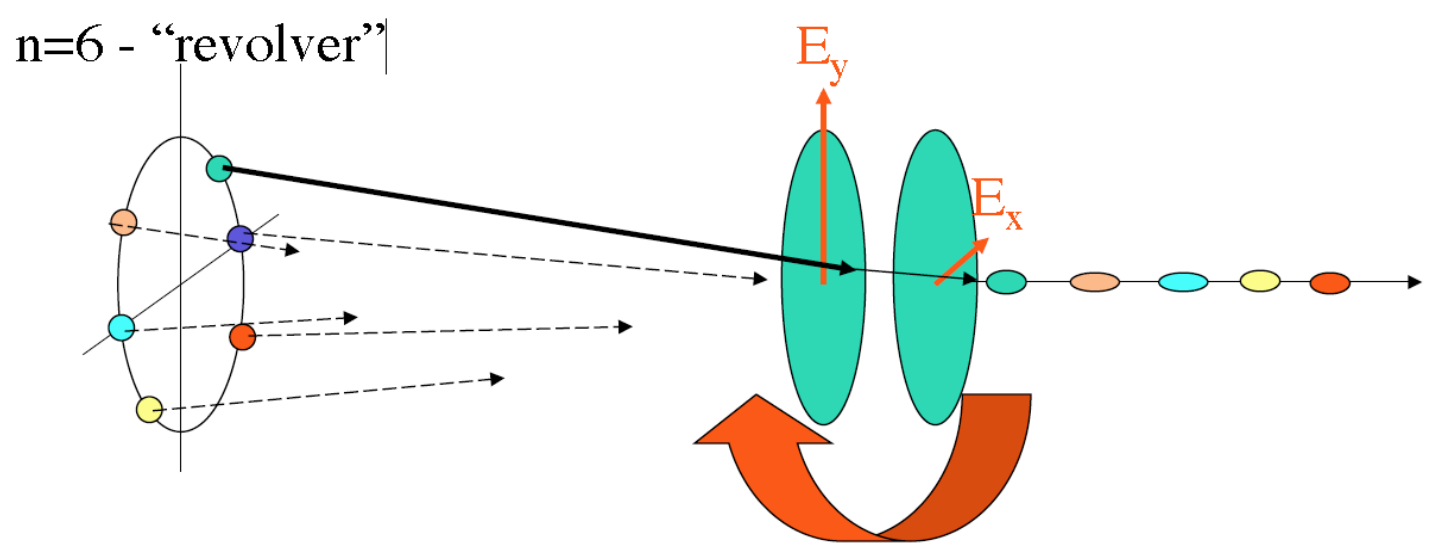

Fig. 2. RF-combiner for $\mathbf{n}$ photo-guns with two RF cavities. RF cavities work with transverse E-mode, $f_{r f}=(1+1 / n) f_{e}\left(x\right.$ and y kick with $90^{\circ}$ shift $)$.

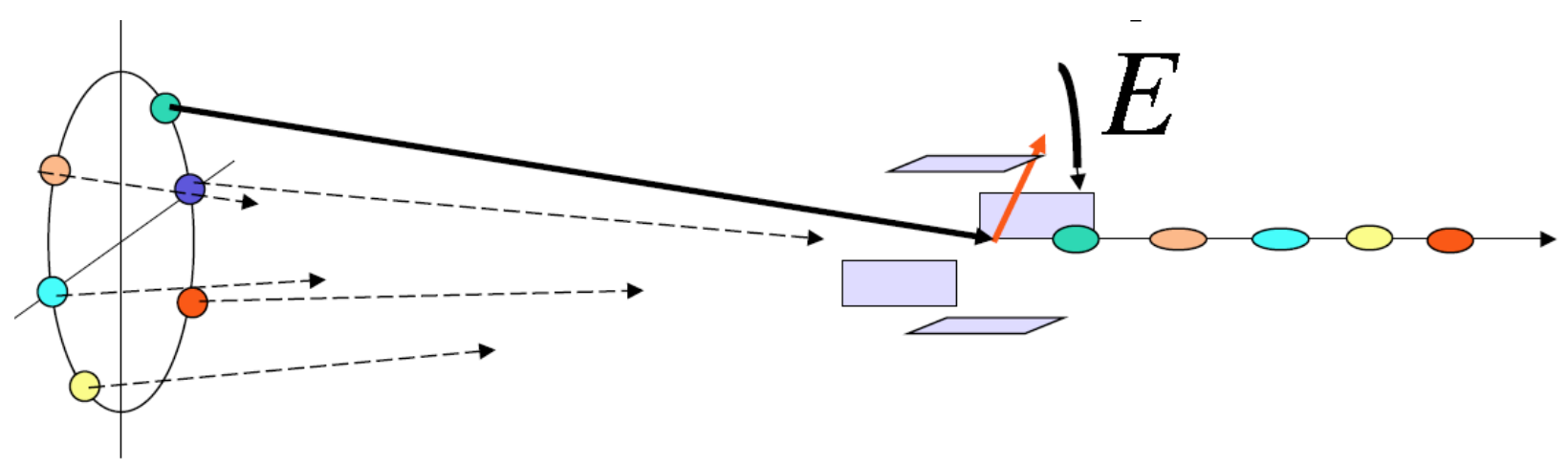

Fig. 3. Low energy of electron from the photo-guns provides for a possibility of using conventional kickers based on strip-lines. This scheme has some advantages compared with the RF-combiner, because both the amplitudes and the voltages applied to the kicker's strip-lines can be easily controlled.

\section{Polarized case}

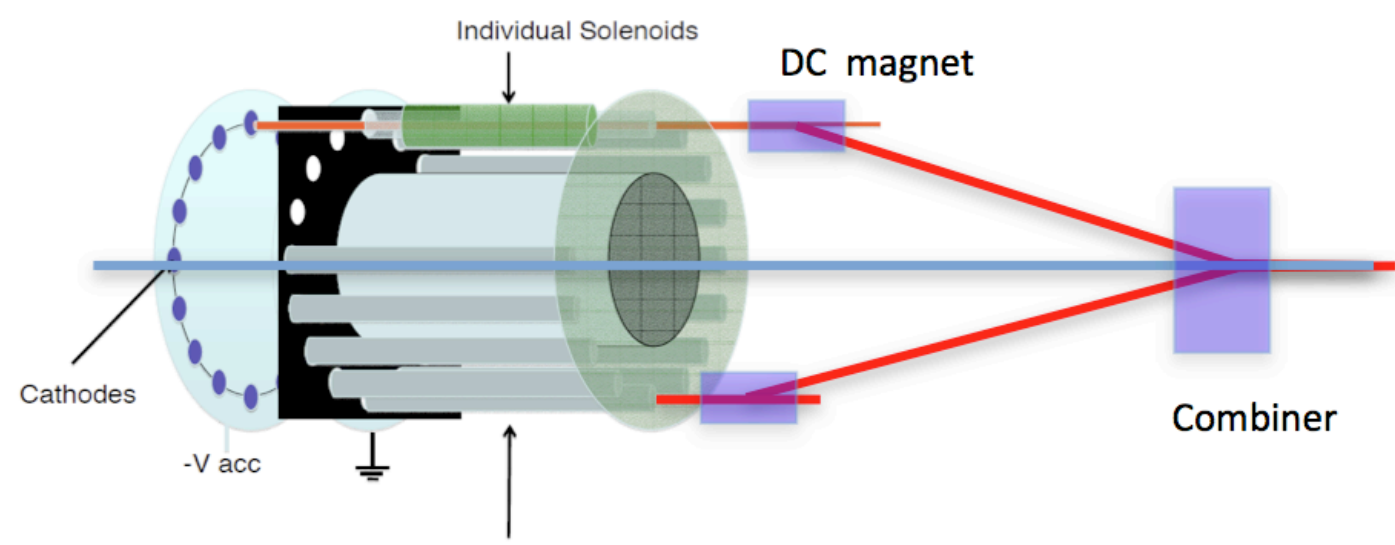

Individual short pipes

Fig. 4. Version of the polarized electron Gatling gun with sixteen photo-cathodes. This specific figure was developed later that the original note. 
Hence spin of electrons can be affected by transverse kick, the system should be modified to have a compensated dogleg trajectories. Such scheme is shown in Fig.4. The beams are extracted from the cathodes along z-axis and then bent by DC magnets towards a combiner. The combiner has rotating magnetic field putting the beams onto the common trajectory.

\section{Conclusion.}

The entire idea of the Gatling gun approach assumes that capabilities of individual cathodes are not affected by the physical presence of other cathodes. This assumption has to be proven experimentally. 